\title{
IS ECONOMIC RECOVERY A MYTH? ROBUST ESTIMATION OF IMPULSE RESPONSES
}

\author{
COEN N. TEULINGS ${ }^{\mathrm{a}}$ AND NIKOLAY ZUBANOV ${ }^{\mathrm{b} *}$ \\ a $C P B$ Netherlands Bureau of Economic Policy Analysis \\ ${ }^{\mathrm{b}}$ Erasmus University Rotterdam, Erasmus School of Economics, Rotterdam, The Netherlands
}

\begin{abstract}
SUMMARY
We estimate the impulse response function (IRF) of GDP to a banking crisis using an extension of the local pro jections method. We demonstrate that, though robust to misspecifications of the data generating process, this method suffers from a hitherto unnoticed bias which increases with the forecast horizon. We propose a correction to this bias and show through simulations that it works well. Applying our corrected local projections estimator to the data from a panel of 99 countries observed between 1974 and 2001, we find that an average banking crisis yields a GDP loss of just under $10 \%$ in 10 years, with little sign of recovery. Like the original local projections method, our extension of it is widely applicable. Copyright (C) 2013 John Wiley \& Sons, Ltd.
\end{abstract}

\section{INTRODUCTION}

The latest financial crisis has left the world economy in a state of disarray, reducing GDP in most OECD countries by an order of magnitude unseen since World War II and the Great Depression. Will output recover from this shock in the medium to long term, or will part of the loss be permanent? While Campbell and Mankiw's (1987) finding of little mean reversion in output suggests that the output loss due to banking crises can be permanent, it does not necessarily have to be so. Since GDP is a mixture of random processes, some of which have a unit root and others do not, the magnitude and duration of the crisis's effect depend on which of these processes are affected. As Paul Krugman wrote in his blog on 3 March 2009, 'I always thought the unit root thing involved a bit of deliberate obtuseness it involved pretending that you didn't know the difference between, say, low GDP growth due to a productivity slowdown like the on that happened from the 1973 to 1995 , on one side, and low GDP growth due to a severe recession.' For Krugman, the claim that banking crises have permanent effects is equivalent to saying that these shocks affect the random walk components of GDP, such as total factor productivity. If a shock affects only the transitory components of GDP, such as the business cycle, it will have a temporary effect disappearing in a few years. The position of the US Council of Economic Advisers (CEA) is similar in spirit to Krugman's: 'A key fact is that recessions are followed by rebound. Indeed, if periods of lower-than-normal growth were not followed by periods of higherthan-normal growth, the unemployment rate would never return to normal.' ${ }^{1}$

Turning to the empirics, the results presented in an article by Cerra and Saxena (2008), which goes under an ominous title, The Myth of Economic Recovery, seem to run contrary to CEA's and Krugman's views. The authors estimate a dynamic model of GDP growth as the function of lagged

\footnotetext{
* Correspondence to: Nikolay Zubanov, Erasmus School of Economics, Erasmus University Rotterdam, PO Box 1738, 3000 DR Rotterdam, The Netherlands. E mail: zubanov@ese.eur.nl

${ }_{1}$ See CEA notes on economic projections and the budget outlook, http://www.whitehouse.gov/administration/eop/cea/ Economic Projections and the Budget Outlook.
} 
growth rates and a dummy for the occurrence of a banking crisis, and use their regression estimates for a recursive calculation of the impulse response function (IRF) of GDP to a banking crisis event. We refer to their estimation procedure as the analytical estimator of the IRF, since it involves calculating the IRF for longer forecasting leads by recursively applying the estimated model to generate a forecast for the intermediate periods. As the title of their paper suggests, the authors find that the effect of a banking crisis on output is large up to 7.5\% of GDP and persists for at least 10 years.

However, as we demonstrate with some examples later in the text, the analytical estimator is sensitive to misspecification of the data-generating process for GDP, the sensitivity of which may have important consequences for the estimated IRF, the more so as the length of the forecast horizon increases. Jorda (2005) proposed a method of estimating IRF that is robust to misspecification of the data-generating process, known as the local projections estimator. Essentially, a local projections estimate of the IRF of variable $y$ to a shock in an exogenous regressor $x k>0$ periods after the shock is the coefficient on $x$ in the regression of $y_{t+k}$ on the regressors measured at time $t$ (i.e. without including their intermediate values realized between $t$ and $t+k$ ). This method is robust to a variety of misspecifications in the underlying model, because instead of using the same set of coefficients for all forecasting horizons a new set of coefficients is estimated for each horizon $k$. This advantage comes at the cost of some loss in the IRF estimates' efficiency, which we quantify for a simple case.

What occupies most of our attention in this study is a hitherto unnoticed bias in the local projections estimator. This bias occurs because in the regression of GDP at $t+k$ on the banking crisis dummy at $t$, which underlies this estimator, there will be observations in which GDP is already affected bythe crisis but the corresponding banking crisis dummy is zero. For an illustration, consider the local projections regression for a forecasting horizon of $k=4$ years. This regression tries to explain GDP at $t+4$ with the variables (lags of GDP and the banking crisis dummy) measured at $t$. Suppose a banking crisis hits the economy only once at time $t_{0}=6$ and will start to have its negative effect on GDP in the same year. Then the GDP levels observed at $t=6,7,8,9$ will contain the effects of a banking crisis from the immediate to 3 years thereafter. However, since the corresponding sets of regressors include the variables observed only in $t-4=2,3,4,5$, the banking crisis occurring at $t_{0}=6$ will not be registered. The negative effects of the crisis in years $t=6,7,8,9$ will thus not be captured by the regressors, and subsequently will be soaked up by the fixed effect, leading to a downward bias in its estimate. The downward bias in the fixed effect's estimate will affect the IRF(4) estimate (the coefficient on the banking crisis dummy), causing a downward bias to it. We provide an analytical expression for the size of the bias in one simple case.

Our solution to correct the above bias is to augment the local projections regression with the dummy variables for the banking crises occurring within the forecast horizon, i.e. between $t$ and $t+k$. Monte Carlo simulations show that, compared to the original local projections estimator, our correction produces IRF estimates closer to the true IRF and more robust to dynamic misspecifications. In fact, since the bias in the local projections method that we identify arises in a variety of settings, our method to eliminate it will see many applications as well.

We run our corrected local projections estimator on a panel of 99 countries observed during 19742001. Our main finding is that an average banking crisis leads to a GDP loss of just under 10\% over a 10-year period (the length of the time series does not allow calculating longer forecasts), and there is little recovery within that period. There is appreciable heterogeneity in the effect of the crisis. African countries suffer greater GDP losses (13\%) over the same 10-year period than non-African countries (7\%). While, qualitatively, our finding that the effects of banking crises are long-lasting is consistent with Cerra and Saxena's (2008), our IRF estimates are larger in magnitude than theirs.

The rest of this paper is organized as follows. Section 2 discusses the analytical and local projections estimators of IRFs. In subsection 2.1, we compare and contrast these two estimators in terms of robustness to misspecifications and efficiency loss. Subsections 2.2 and 2.3 discuss the the local projections estimator's bias and our method to eliminate it. Subsection 2.4 contains Monte Carlo simulations 
illustrating the bias performance of the corrected local projections estimator in comparison with other estimators. In Section 3 we apply the corrected local projections estimator to the data on banking crises in order to estimate their effect on GDP. Section 4 concludes.

\section{IRF ESTIMATION IN MODELS WITH FIXED EFFECTS}

\subsection{Analytical and Local Projections Estimators}

Consider the following trend-stationary $\operatorname{AR}(R)$ panel data model with fixed-effects $\alpha_{0 i}$ and a common linear time trend with slope $\alpha_{0}^{*}$ :

$$
y_{i, t}=\alpha_{0 i}+\alpha_{0}^{*} t+\sum_{r=1}^{R} \alpha_{1 r} y_{i, t} r+\sum_{l=0}^{L} \alpha_{2 l} d_{i, t} l+u_{i, t}
$$

where $y_{i, t}$ is $\log$ GDP per capita for country $i=1 \ldots N$ in year $t=1 \ldots T, d_{i, t}$ is a dummy variable equal to 1 if a banking crisis in country $i$ starts in year $t$ and 0 otherwise, and $u_{i, t} \sim N\left(0, \sigma^{2}\right)$ is the idiosyncratic error term, independent of the regressors and identically distributed for each country and year.

The stationarity assumption implies $\left|\sum_{r=1}^{R} \alpha_{1 r}\right|<1$. We further assume that banking crises arrive randomly at a country-specific rate $\lambda_{i}$, so that $\mathrm{E}\left[d_{i, t}-\lambda_{i}\right]=0,{ }^{2}$ and that banking crises occur independently of all forward and backward realizations of the error term, i.e. $\mathrm{E}\left[u_{i, s} d_{i, t}\right]=0, \forall s, t$. Hence we assume that the process determining the occurrence of banking crises is exogenous to the process that drives GDP. This is a strong assumption, which we partly test in Section 3.3.

Define the IRF of GDP to banking crisis $k$ years after its start as

$$
\operatorname{IRF}(k) \equiv \mathrm{E}\left[y_{i, t+k} \mid d_{i, t}=1, y_{i, s}, d_{i, s}, s<t\right]-\mathrm{E}\left[y_{i, t+k} \mid d_{i, t}=0, y_{i, s}, d_{i, s}, s<t\right]
$$

Based on this definition, one can calculate the IRF analytically for each $k$ by expressing the conditional expectation of $y_{i, t+k}$ as a function of equation (1)'s parameter estimates. For example, for $R=1$ and $L=0$, $\operatorname{IRF}(k)=\alpha_{20} \alpha_{11}^{k}$. This method of calculating IRF, which we refer to as the analytical estimator, has two problems. First, it relies entirely on the coefficients of the underlying model (1) and therefore its consistency depends on this model's correct specification. As the model includes more lags of $y_{i, t}$ and $d_{i, t}$, and as the length of the forecast $k$ increases, $\operatorname{IRF}(k)$ becomes a complex expression that is increasingly sensitive to even slight specification errors. Since we are interested in the medium- to long-run effect of a banking crisis, the growing sensitivity of IRF estimates to misspecifications is a serious problem. We will offer some examples of how misleading IRF estimates based on misspecified models can be. Second, the growing complexity of $\operatorname{IRF}(k)$ will make the calculation of its standard errors increasingly cumbersome, and the distribution of the parameter increasingly fat-tailed, since it raises the underlying coefficient to progressively higher powers (e.g. $a_{11}^{k}$ in the example above).

An alternative to calculating the IRF analytically which is robust to dynamic misspecifications is known as the local projections estimator (Jorda, 2005). This estimator, initially developed for the case of a vector autoregressive (VAR) model, is generally applicable and has recently been extended to the case of a nonstationary VAR (Chong et al., 2012). Here we consider its application for a single-equation model. The local projections method estimates $\operatorname{IRF}(k)$ directly from the forecast equation for GDP $k$

\footnotetext{
${ }^{2}$ Cerra and Saxena (2008, pp. 448 456) provide some evidence to support this assumption.
} 
periods ahead, as we now explain. Since the process is stationary, the lagged values of $y_{i, t}$ can be eliminated from the model by recursive substitution of lagged versions of equation (1) to obtain

$$
y_{i, t}=\gamma_{0 i}+\gamma_{0}^{*} t+\sum_{l=0}^{\infty} \gamma_{2 l} d_{i, t} l+\sum_{m=1}^{\infty} \gamma_{3 m} u_{i, t} m+u_{i, t}
$$

where the $\gamma$-parameters are functions of the $\alpha$-parameters. ${ }^{3}$ Hence $y_{i, t}$ can be expressed as a linear function of all past shocks $d_{i, t}$ and $u_{i, t}$.

The $k$-period-ahead forecast of GDP conditional on the information available at $t$ is

$$
\mathrm{E}\left[y_{i, t+k} \mid y_{i, s}, d_{i, s}, s \leq t\right]=\mathrm{E}\left[y_{i, t+k} \mid u_{i, s}, d_{i, s}, s \leq t\right]
$$

(the second equality uses equation (3) to eliminate the lags of $y_{i, t}$ from equation (1)). Since, by assumption, $\mathrm{E}\left[d_{i, s}\right]=\lambda_{i}$ and $\mathrm{E}\left[u_{i, s}\right]=0$, the conditional forecast satisfies

$$
\mathrm{E}\left[y_{i, t+k} \mid y_{i, s}, d_{i, s}, s \leq t\right]=\gamma_{0 i k}+\gamma_{0}^{*}(t+k)+\sum_{l=k}^{\infty} \gamma_{2 l} d_{i, t+k} l+\sum_{m=k}^{\infty} \gamma_{3 m} u_{i, t+k} 1 \quad m
$$

where $\gamma_{0 i k}=\gamma_{0 i}+\sum_{l=0}^{k 1} \gamma_{2 l} \lambda_{i}$. The shocks hitting $y_{i, t}$ between time $t$ and time $t+k$ do not affect its conditional forecast (equation (4)) since their expected value, conditional on the information available at the moment that the forecast is made, is zero. By the definition of $\operatorname{IRF}$ (equation (2)), $\operatorname{IRF}(k)=\gamma_{2 k}$. This substitution procedure can be reversed by solving equation (1) for $u_{i, t}$ and using that solution to eliminate $u_{i, t}$ and its lags from equation (4), giving

$$
\mathrm{E}\left[y_{i, t+k} \mid y_{i, s}, d_{i, s}, s \leq t\right]=\delta_{0 i k}+\delta_{0 k}^{*} t+\sum_{r=1}^{R} \delta_{1 r k} y_{i, t} r+\sum_{l=0}^{L} \delta_{2 l k} d_{i, t} l
$$

where the $\delta$-parameters can be expressed as functions of the $\gamma$-parameters in the original forecast equation. These expressions are of little interest here, except for one:

$$
\delta_{20 k}=\gamma_{2 k}=\operatorname{IRF}(k)
$$

which is estimated from the conditional forecast equation (5) specifically for each $k$, by running

$$
y_{i, t+k}=\delta_{0 i k}+\delta_{0 k}^{*} t+\sum_{r=1}^{R} \delta_{1 r k} y_{i, t} r+\sum_{l=0}^{L} \delta_{2 l k} d_{i, t} l+v_{i, t+k}
$$

where

$$
v_{i, t+k}=\sum_{l=0}^{k} \gamma_{2 l}\left(d_{i, t+k} l-\lambda_{i}\right)+\sum_{m=1}^{k 1} \gamma_{3 m} u_{i, t+k} m+u_{i, t+k}
$$

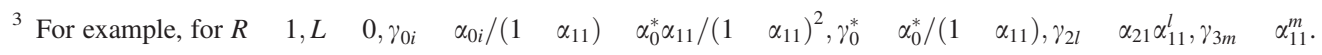


Jorda's local projection method can be interpreted as estimating a reduced-form model, where all endogenous variables the realizations of $y_{i, s}$ between the moment $t$, at which the forecast is made, and the moment $t+k$ have been eliminated. The forecast error, following a moving average process of order $k-l$, is serially correlated and requires an appropriate general least squares transformation of the original equation to achieve full efficiency of the estimator. Even then there will be an efficiency loss compared to the analytical estimator, since estimating the local projections equation (6) for forecast horizon $k$ requires, in effect, an extra $k$ lags of the regressors, which is increasingly taxing as $k$ gets larger. A simpler alternative to specifying the moving average process in the residuals is to use ordinary least squares (OLS) with serial correlation-robust standard errors. This is what we do. Proposition 1 (see web appendix (supporting information) for a full proof), gives an estimate of the efficiency loss as compared to the analytical IRF estimator for one specific case.

Proposition 1. Consider model (1) with $\alpha_{0 i}=\alpha_{0}^{*}=0, R=1$ (i.e. $\mathrm{AR}(1)$ without trend and intercept) and $L=-1$ (i.e. no banking crises). The variance of the analytical estimator of $\operatorname{IRF}(k)$ to a unit shock in the error term, $\hat{\alpha}_{11}^{k}$, satisfies

$$
\left.\operatorname{plim}\left(N(T-1) \operatorname{var}\left[\hat{\alpha}_{11}^{k}\right]\right)=k^{2}\left(1-\alpha_{11}^{2}\right) \alpha_{11}^{2(k} 1\right)
$$

The variance of the local projections estimator of the same $\operatorname{IRF}(k), \widehat{\delta}_{11 k}$, satisfies

$$
\operatorname{plim}\left(N(T-k) \operatorname{var}\left[\widehat{\delta}_{11 k}\right]\right)=1-\alpha_{11}^{2 k}+2(k-1)-2 \frac{\alpha_{11}^{2}-\alpha_{11}^{2 k}}{1-\alpha_{11}^{2}}
$$

As a graphic illustration, Figure 1 plots the probability limits of variances of the analytical $\left(\hat{\alpha}_{11}^{k}\right)$ and local projections $\left(\hat{\delta}_{11 k}\right)$ IRF estimates (the expressions in Proposition 1 above) and their ratio for $\alpha_{11}=0.95$ for $k$ ranging from 1 to 10 . The efficiency loss of the local projections estimator relative to analytical IRF estimator, measured as the ratio of the probability limits of the variances, is growing with $k$, reaching almost 2 for $k=10$. This loss of efficiency is counterbalanced by a greater robustness of the local projections estimator, which we now illustrate with the following two examples.

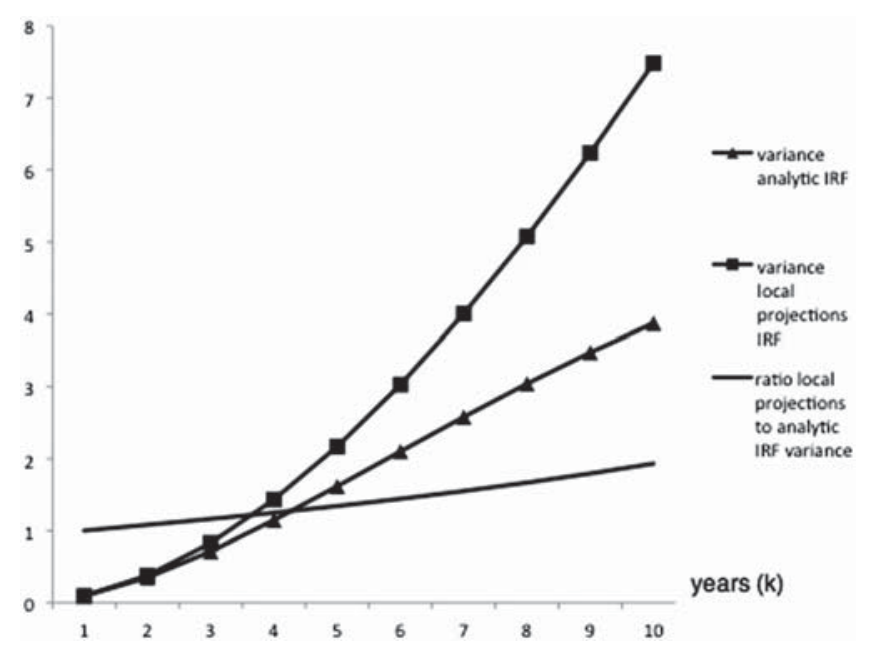

Figure 1. The probability limits of variances of the AR(1) process IRFs estimated analytically and through local projections 


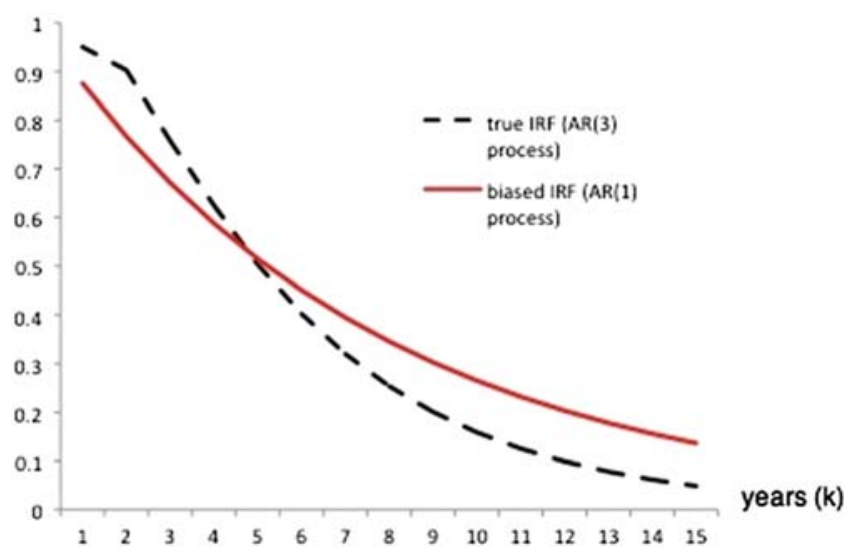

Figure 2. The IRFs from the AR(3) and AR(1) models

Example 1. Consider a third-order autoregressive model (1) where $\alpha_{0 i}=\alpha_{0}^{*}=0$ (no trend, no fixed effect), $R=3$ and $L=-1$ (again, no banking crisis dummy). Suppose that the coefficient for the second lag of the dependent variable is zero, $\alpha_{12}=0$, and that the coefficient for the third lag, $\alpha_{13}$, is so much smaller in magnitude than $\alpha_{11}$ that its regression estimate is statistically insignificant, so that the econometrician decides to proceed with the parsimonious $\mathrm{AR}(1)$ specification. This misspecification will result in a biased estimate for the $\mathrm{AR}(1)$ parameter $\alpha_{11}$, since the probability limit of its OLS estimate is equal to

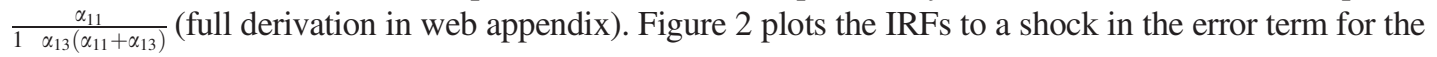
true AR(3) model and the estimated AR(1) model for the case of $\alpha_{11}=0.95$ and $\alpha_{13}=-0.10$. In this case, if one proceeds with the misspecified AR(1) process one gets a biased $\alpha_{11}=0.88$ and consequently an IRF which underestimates the shock for relatively small $k$ and overestimates it for larger $k$. On the other hand, applying the local projections method yields a consistent IRF even for the incorrectly chosen $R=1$, since it is estimated separately for each $k$ so as to minimize the difference between the actual and predicted value of $y_{i, t+k}$ for each $k$, irrespective of the assumed specification of the data-generating process. ${ }^{4}$

Example 2. Consider the implications of the restriction for the shocks in $d_{i, t}$ and $u_{i, t}$ to have the same dynamics, which is implicit in the previous example and Proposition 1. This implicit restriction will result in a bias in the analytical estimator of the IRF in the presence of unobserved dynamic components in $y_{i, t}$, as we now explain. Suppose $y_{i, t}$ is the sum of two components: (i) $x_{i, t}$, which is the effect of banking crisis that dies out after a year; and (ii) $z_{i, t}$, which is an unobserved AR(1) process. Hence

$$
\begin{aligned}
& y_{i, t}=x_{i, t}+z_{i, t}, \\
& z_{i, t}=\gamma z_{i, t} \quad 1+u_{i, t}, u_{i, t} \sim N\left(0, \sigma^{2}\right) \\
& x_{i, t}=\alpha_{21}\left(d_{i, t}-\lambda\right)
\end{aligned}
$$

The presence of $z_{i, t}$ induces autocorrelation in $y_{i, t}$, and if $z_{i, t}$ is unobserved the AR(1) coefficient in model (1) on $y_{i, t} \quad$ (under the simplifying restriction $\alpha_{0 i}=\alpha_{0}^{*}=0$ ) satisfies (full derivation in web appendix):

$$
\operatorname{plim}\left(\widehat{\alpha}_{11}\right)=\frac{\gamma}{1+\lambda(1-\lambda) \alpha_{21}^{2}\left(1-\gamma^{2}\right) \sigma^{2}}
$$

\footnotetext{
${ }^{4}$ The advantage of applying the local projections method to the correctly specified model (with $R \quad 3$ in our example) is efficiency gain, although this gain may be offset by the ensuing loss of observations.
} 
Consequently, a banking crisis will appear to have the same dynamic effect as any shock to $z_{i, t}$ through the $\operatorname{AR}(1)$ term $\alpha_{11}$, whereas in fact its effect is one-off. ${ }^{5}$ The analytical IRF estimator is clearly prone to this error. The local projections estimator, on the other hand, does not suffer from it because it estimates the IRF (the coefficient $\delta_{20 k}$ ) directly from the local projections equation (6), without relying on the (mis)estimated autoregression coefficients as does the analytical IRF estimator. Thus, estimating equation for the model in this example, even misspecifying its dynamic structure with $R=L=1$, will $\operatorname{give} \operatorname{plim}\left(\hat{\delta}_{20 k}\right)=\alpha_{21}$ for $k=0$, and zero for $k \geq 1$. Since the local projections estimator is robust to the misspecification illustrated in the above examples, albeit at the expense of some loss in efficiency, this estimator gives a more reliable picture of the true IRF.

\subsection{The Bias in the Local Projections Estimator}

Regrettably, the local projections estimator presented above is subject to a hitherto unnoticed bias which increases with the forecast horizon $k$. Suppose a country $i$ is hit by a banking crisis only once at, say, $t=6$, so $d_{i, 6}=1$ and $d_{i, t}=0$ for all other $t$, and that the banking crisis starts to have an effect in years $t=6$ onwards. Let us assume, for the ease of exposition, that $R=0$ (no lagged dependent variables) and $\alpha_{0}^{*}=0$ (no trend). Consider what happens when the researcher applies the local projections estimator to estimate $\operatorname{IRF}(k)$. For $k=0$, there is no problem, since regressing $y_{i, t}$ on $d_{i, t}$ and a country-specific constant capturing the fixed effect will produce an unbiased estimate of the immediate effect of the banking crisis, $\operatorname{IRF}(0)$.

The estimator will be biased for $k \geq 1$ because for some $t$ s the banking crisis will fall between $t$ and $t+k$ and thus will not be part of the regression equation, causing misspecification. Suppose $k=4$. The local projectionsregression of $y_{i, t+4}$ on $d_{i, t}$ and a fixed effect is the right specification for observations at $t=0$ and $t=1$, since with $d_{i, 0}=d_{i, 1}=0, y_{i, t+4}$ is just equal to the country fixed effect (the banking crisis will occur only at $t=6$ ). However, for $t=2, y_{i, t+4}=y_{i, 6}$ is already affected by the banking crisis at $t=6$, but this event has not yet been registered in the regressors observed at $t=2$. Therefore, $y_{i, 6}$ is misspecified because in fact it is equal to the fixed effect plus $\operatorname{IRF}(0)$ rather than just the fixed effect that the local projections equation will estimate. Similarly, there is a misspecification for $t=3$, when $y_{i, t+4}=y_{i, 7}$ equals the fixed effect plus $\operatorname{IRF}(1)$, for $t=4$, when $y_{i, t+4}=y_{i, 8}$ equals fixed effect plus $\operatorname{IRF}(2)$, and for $t=5$ when $y_{i, t+4}=y_{i, 9}$ equals fixed effect plus IRF(3). From $t=6$ onwards, we are back to the correct specification for $y_{i, t+4}$ since the banking crisis at $t=6$ is now registered in the observations. Note that including lags of $y_{i, t}$ as additional regressors in the local projections equation will not help, since, dated at $t-1$ or earlier, they will not bring any information about the banking crisis occurring between $t$ and $t+k$ which is causing the bias.

The above example is illustrated in Figure 3, which plots actual (solid line) versus specified (dashed line) dependent variable in the local projections regression for $k=4$ of $y_{i, t+4}$ on the lags of $d_{i, t}$ and the constant term equal to 1 , assuming $\operatorname{IRF}(k)=-0.8^{k}$ so $\operatorname{IRF}(4)=-0.41$. The difference between the actual and (mis)specified $y_{i, t+4}$, equal to $\operatorname{IRF}(0)+\operatorname{IRF}(1)+\operatorname{IRF}(2)+\operatorname{IRF}(3)$ (the size of the shaded area A in the upper graph of Figure 3), will be absorbed by the regression's error term. Then, since the firstorder condition for the constant term sets the mean regression error to zero, this difference will be spread evenly to all observations, resulting in a downward bias to the estimate of the constant term (or fixed effect for panel data) of $[\operatorname{IRF}(0)+\operatorname{IRF}(1)+\operatorname{IRF}(2)+\operatorname{IRF}(3)] / T$, as illustrated in the lower graph of Figure 3 . For the assumed $\operatorname{IRF}(k)$ and the length of observations $T=20$, the bias to the fixed effect's estimate is -0.15 . This bias translates one-for-one into the bias for estimate of IRF(4), as we show in the following Proposition 2 (proof in web appendix), for the case without time trend and lags of the dependent variable that we considered above.

\footnotetext{
${ }^{5}$ Cai and Den Haan (2009) make the same point criticizing 'one type shock' models of output which tend to overestimate the long run impact of recessions.
} 

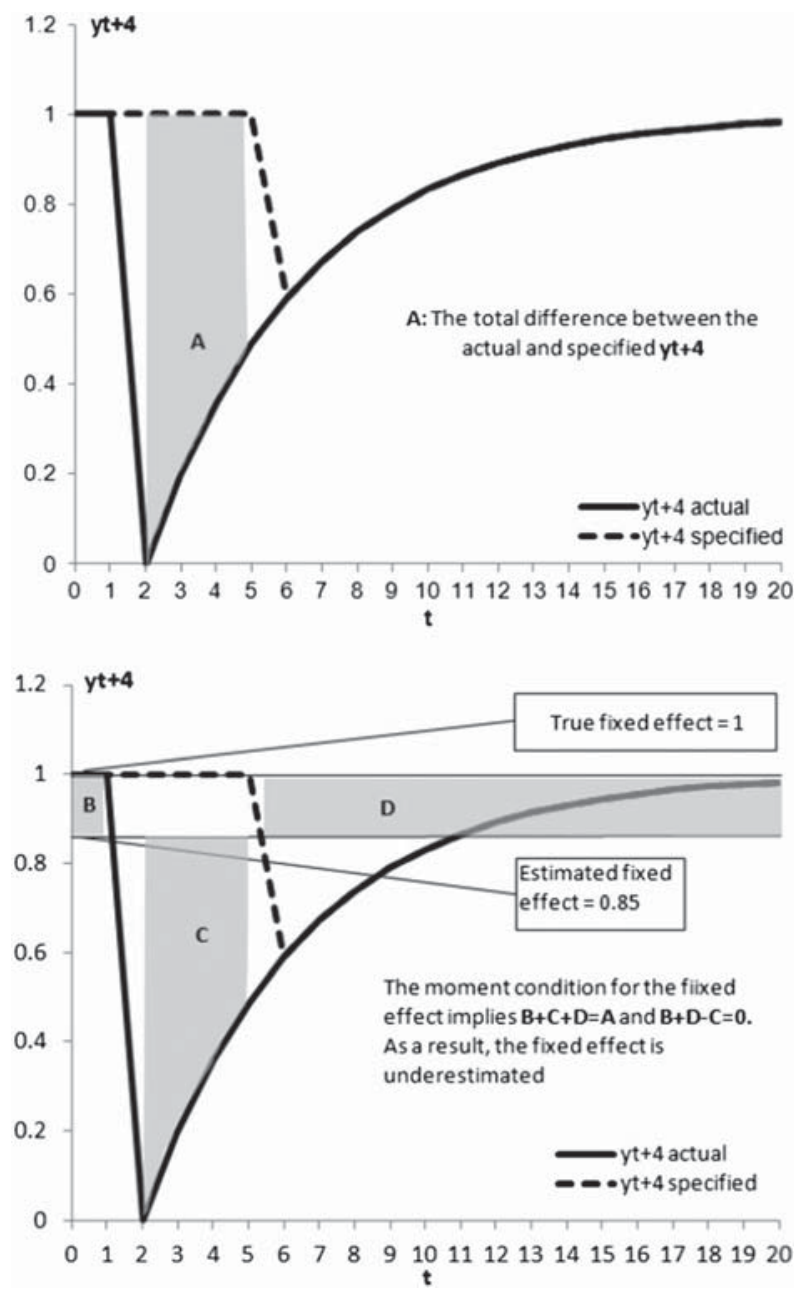

Figure 3. Actual versus specified dependent variable $\left(y_{t+4}\right)$ in the local projections regression for $k=4$

Proposition 2. Consider the local projections equation (6) with $R=\alpha_{0}^{*}=0$ applied on a sample of countries which are hit by a banking crisis at most once and not in the first $k$ years of the period of observation. Let data be available for estimation for $T$ years (so the length of observations is $T+L+k$ years). Then the biases of the local projections estimates of the fixed effect $\left(\delta_{0 i k}\right)$ and $\operatorname{IRF}(\mathrm{k})\left(\delta_{21 k}\right)$ satisfy $^{6}$

$$
\begin{gathered}
\mathrm{E}\left[\widehat{\delta}_{0 i k}-\delta_{0 i k}\right]=(T-L){ }^{1} \sum_{l=0}^{k} \gamma_{2 l}, \\
\mathrm{E}\left[\widehat{\delta}_{21 k}-\delta_{21 k}\right]=-(T-L)^{1} \sum_{l=0}^{k} \gamma_{2 l}
\end{gathered}
$$

\footnotetext{
${ }^{6}$ Including the trend and/or lags of the dependent variable will not change the message of Proposition 2, but make the maths less transparent without adding further insights. The assumptions that the crisis occurs at most once in a given country, and not in the first $k 1$ years of observations, were introduced to simplify the mathematics. They are not crucial for the essentials of our argu ment. Since banking crises are assumed to be exogenous in our model, excluding countries not satisfying these assumptions will not result in a sample selection problem.
} 
Proposition 2 implies a downward bias (in absolute magnitude) of the local projections IRF estimate for $k \geq 1$, which increases with the forecast horizon $k$, both because the cumulative effect of the banking crisis, $\Sigma_{l=0}^{k} 1 \gamma_{2 l}$, omitted from the regression becomes larger, and because the effective number of observations $T$ becomes smaller, since more data are lost when regressing $y_{i, t+k}$ on the variables measured at $t$. The bias also increases with $L$, because the estimated coefficient $\widehat{\delta}_{21 k}$ will have to adjust more to offset the bias in the estimated fixed effect $\widehat{\delta}_{0 i k}$ for $L$ of the $T$ observations available. Note that the part of the argument in Proposition 2 concerning the bias to the IRF estimates applies in both time series and panel settings; in particular, the size of this bias does not depend on the fraction of countries in the sample that have been hit by a banking crisis.

\subsection{An Alternative Estimator}

Our solution to the bias in the local projections estimator is to correct it by including in the local projections equation (6) the banking crises occurring between $t$ and $t+k$. The thus corrected local projections equation is

$$
\begin{aligned}
y_{i, t+k} & =\delta_{0 i k}+\delta_{0 k}^{*} t+\sum_{r=1}^{R} \delta_{1 r k} y_{i, t} r+\sum_{l=0}^{L} \delta_{2 l k} d_{i, t} l+\sum_{l=0}^{k} \gamma_{2 l} d_{i, t+k} l+v_{i, t+k}^{*}, \\
v_{i, t+k}^{*} & =\sum_{m=0}^{k} \gamma_{3 m} u_{i, t+k} m+u_{i, t+k}
\end{aligned}
$$

where $v_{i, t+k}^{*}$ is the error term analogous to the term $v_{i, t+k}$ in the local projections equation (6) but conditional on the information available at time $t$ and the information on the occurrence of banking crises between $t$ and $t+k$. Including the intermediate banking crises in regression (8) produces unbiased estimates of $\operatorname{IRF}(k)$, since the error term no longer contains $d_{i, t}$.

The idea of augmenting the forecast equation with intermediate observations has been applied before, albeit in a different setting, by Faust and Wright (2011). They show that the accuracy of a forecast of excess bond and stock returns improves when the model is augmented with forecast errors observed ex post between $t$ and $t+k$. To relate their argument to our case, adding intermediate banking crises to the model reduces the local projections IRF's standard errors through a reduction in the variance of the error term $v_{i, t+k}$ in equation (6); see equation (7). However, specifically for our case, adding dummies for intermediate banking crises not only improves efficiency but also takes away the bias in the local projection estimator.

So far we have ignored the bias due to negative correlation between the error term and lagged values of $y_{i, t}$, described in Nickel (1981). This bias is induced by the fixed-effects or first-difference transformation, and leads to downward-biased estimates of the coefficients $\delta_{1 r k}$ on the lags of $y_{i, t}$. If there is negative correlation between GDP and banking crises, the Nickell bias also leads to underestimated IRF coefficients $\delta_{20 k}$. The strength of this bias decreases as $T$ grows large relative to $N$. While an Arrelano Bond type estimator is the most often used method to correct for the Nickell bias, an alternative unbiased IRF estimator can be derived from a slightly rewritten version of equation (8) in which all lags of $y_{i, t}$ are eliminated through recursive substitution (as in equation (4)):

$$
y_{i, t}=\gamma_{0 i k}+\gamma_{0 !} t+\sum_{l=0}^{\infty} \gamma_{2 k} d_{i, t} l+v_{i, t}
$$

In addition to being free from the Nickell bias, the estimator based on equation (9) is convenient to apply as it produces all $\gamma_{2 l}, l=0, k$, at once. The disadvantage of this estimator, however, is lower 
efficiency compared to the (corrected) local projections one, which is due to the loss of lags of $y_{i, t}$. Besides, turning to its feasible version, there is a question about the number of lags of the banking crisis dummy to be included in equation (9), which ultimately is an empirical question. In our analysis, we observe that the estimated $\gamma_{2 l}$ s stabilize when the number of lags is 18 or more.

\subsection{Monte Carlo Simulations of the Bias in the IRFs}

Here we present stochastic simulation results for the bias in the IRF estimates obtained from the three estimators that we have discussed: (i) the analytical estimator applied by Cerra and Saxena (2008); (ii) Jorda's (2005) local projections estimator based on equation (6), which does not include banking crises between years $t$ and $t+k$; and (iii) the corrected local projections estimator based on equation (8) which includes these banking crises. We generated 1000 counterfactual datasets based on the following AR(5) process:

$$
\begin{aligned}
& y_{i, t}=\alpha_{0 i}+0.25 y_{i, t \quad 1}+0.8 y_{i, t}{ }_{2}+0.4 y_{i, t \quad 3}-0.1 y_{i, t} 4-0.5 y_{i, t} 5 \\
& -0.035 d_{i, t \quad 1}-0.045 d_{i, t 2}-0.03 d_{i, t 3}-0.01 d_{i, t} 4-0.01 d_{i, t} 5+u_{i, t}
\end{aligned}
$$

where the fixed effects $\alpha_{0 i} \sim U(0,3), d_{i, t}$ is equal to 1 if $\alpha_{0 i} / 5+3 U(0,1)<0.45$, and zero otherwise, and the idiosyncratic error term $u_{i, t}$ follows a standard normal distribution. Note that in model (10) the contemporaneous effect of banking crisis on output is assumed to be zero and thus the local projections bias occurs for $k>1$.

For each of the 1000 datasets, 100 observations of $y_{i, t}$ were generated for $t=1,100$, after which the first 70 data points were discarded to avoid $y_{i, t}$ being determined by the initial, randomly chosen values. Hence each dataset had dimensions corresponding to the real data, with $T=30$ and $N=100$ (see Section 3.1). The parameters of equation (10) have been chosen so that the moments of the generated data are similar to those in the real data. Thus the frequency of banking crises is about 0.05 , the correlation between the country fixed effect $\alpha_{0 i}$ and the banking crisis dummy $d_{t}$ is about -0.2 , and the analytical IRF that one would obtain in the perfect knowledge of the parameters of (10) is close to the one that we actually estimate on our data (see Section 3.2).

We then estimated a selection of empirical specifications of equation (10) with different numbers of lags of $y$ and $d$, as well as their corresponding forecast equations up to $k=10$, on each resulting dataset using the fixed effects estimator with serial correlation-robust standard errors. Figure 4 plots for each of the three estimators the means of the IRFs estimated in each of the 1000 runs on different assumed specifications of the data-generating process (10), as well as the true IRF implied by it. The results for the analytical estimator are widely different from the true IRF. Parsimonious specifications produce much underestimated results on the entire length of the forecast horizon. Only for the number of lags of $y$ and, especially, $d$ close to their true value of 5, do the analytical IRF estimates converge to the true IRF. The analytical estimator is particularly sensitive to the number of lags of $d$ included in the estimated regression equation. The consequences of this sensitivity are important, because longer lags of the banking crisis dummy are increasingly likely to be excluded from the specification by an econometrician who strives for a parsimonious model. Thus our simulations (results available on request) show that the estimated $t$-statistics of further lags of $d$ are progressively smaller and further apart from their true levels as implied by the data generation process we specified.

The second method Jorda's (2005) original local projections estimator produces IRFs that are much more robust to misspecification and less biased. However, while the bias of the analytical estimator decreases as the empirical model's specification approaches the correct one (equation (10)), the bias in this version of the local projections estimator remains appreciable, and increases towards the end of the forecast horizon as our theory predicts. Our corrected local projections estimator, which includes intermediate banking crisis observations, produces the best results. Its estimated IRFs are 


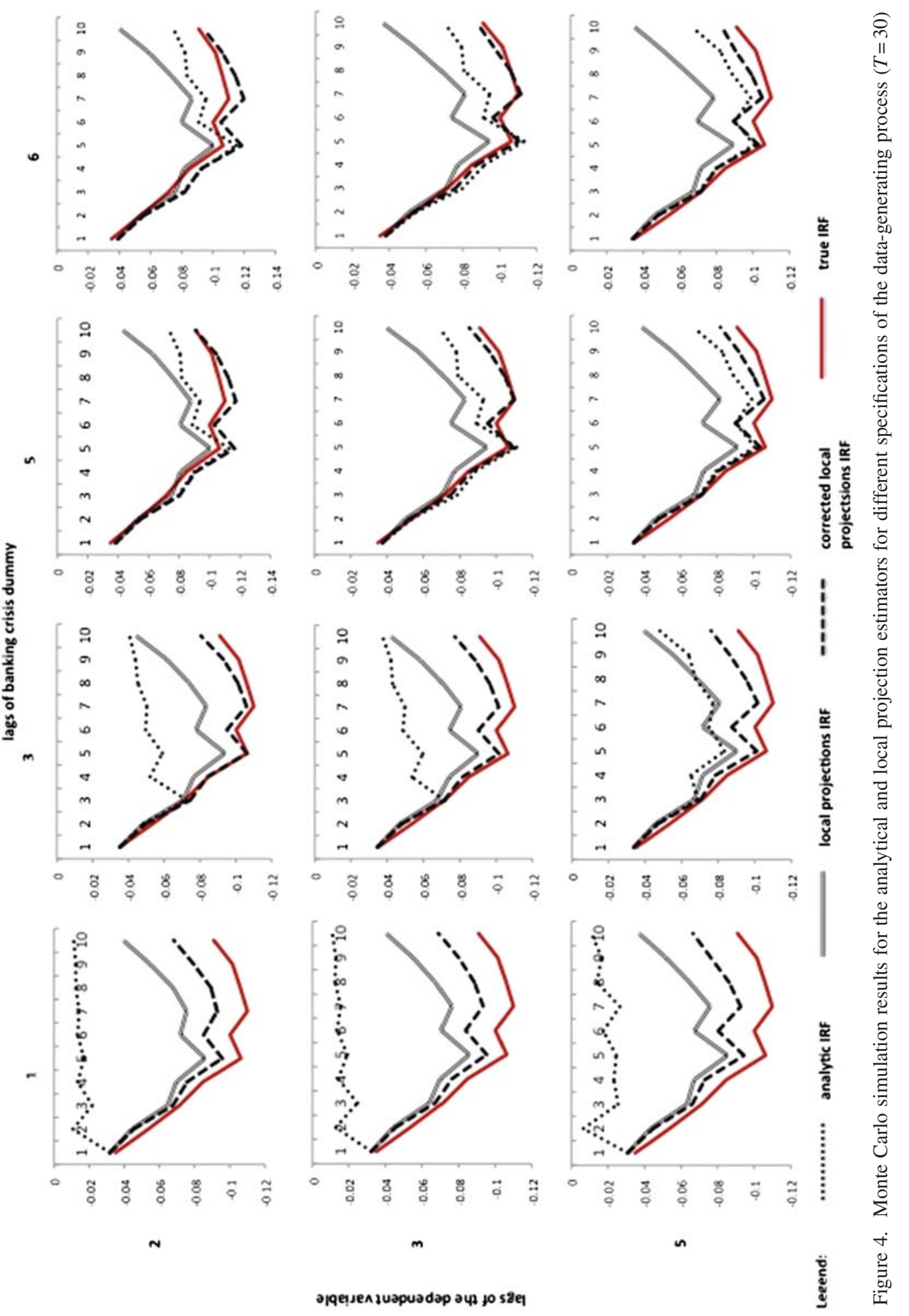


robust to even serious misspecifications of the model, and its bias is smaller than that of the other two estimators. Hence our simulations support our theoretical arguments laid out in Sections 2.1 and 2.2.

All three estimators are sensitive to the Nickell bias, which will occur as long as there is persistency in the dependent variable. Although this bias can be large in finite samples (see Judson and Owen, (1999) and Alvarez and Arellano, (2003) for simulation results), it disappears as panels become longer. One might therefore be interested in gauging how this bias affects our IRF estimates at different lengths of the available time period holding the intertemporal correlation in the dependent variable (i.e. the coefficients on the lags of $y_{i, t}$ ) constant. For this purpose, we simulated the IRFs obtained through two methods the analytical estimator and the corrected local projections estimator applied to panels of different lengths: $T=20$, 30, 40, 50 and 100. The results are plotted in Figure 5. For each estimator, we estimate one misspecified model (three instead of five lags for both $y_{i, t}$ and $d_{i, t}$ ), the IRF from which we plot with a dashed line and one correctly specified model, plotted with a solid line. As expected, the bias is rather large for $T=20$ but declines when $T$ goes up. The IRFs estimated with our corrected local projections estimator (the upper part of Figure 5) are close to the true IRF starting from $T=30$ and are remarkably robust to model misspecifications. The analytical IRF estimates (the lower part of Figure 5) converge to the true IRF as well, but do so more slowly than corrected local projection IRFs. More importantly, unlike the corrected local projections, the analytical IRFs converge to the true IRF only when the model is correctly specified.

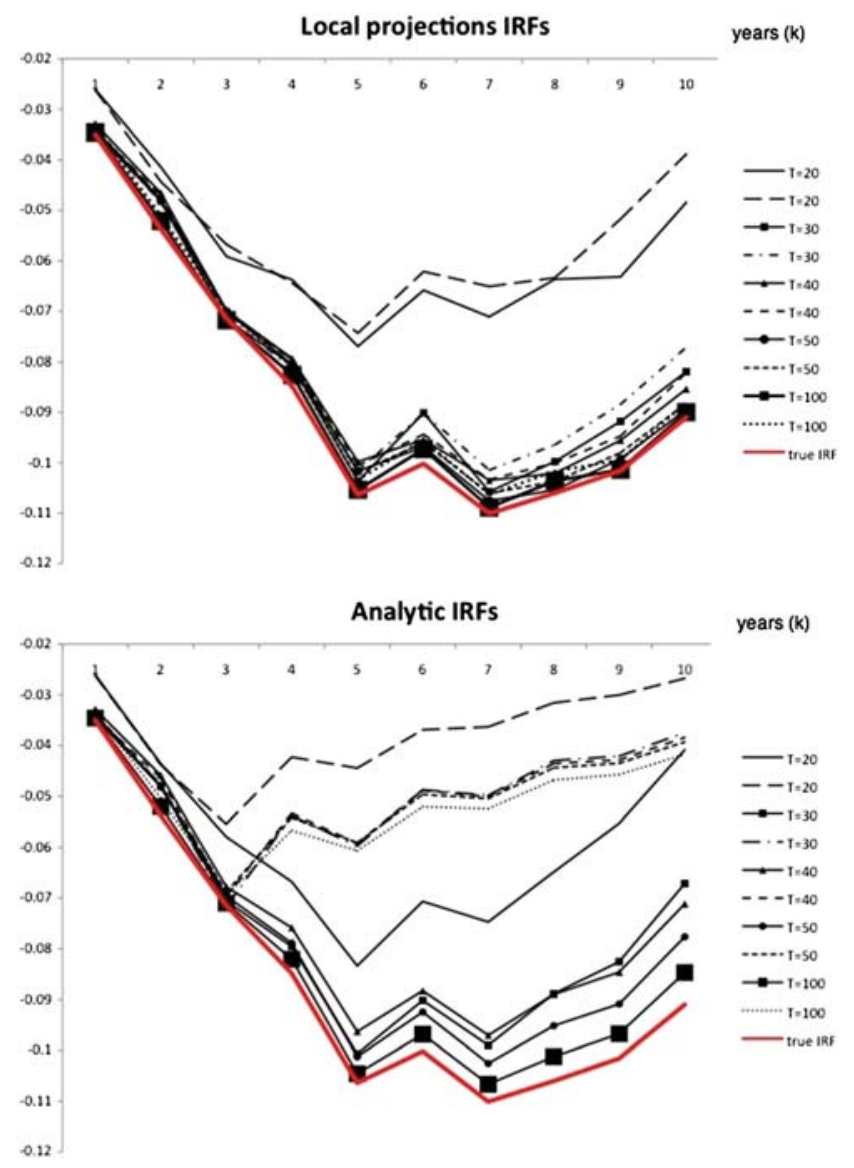

Figure 5. Simulated corrected local projections and analytical IRFs for different lengths of observations, $T$. Solid lines denote IRFs from correctly specified model, and dashed lines denote IRFs from a misspecified model 
Table I. Impulse response estimates for the effect of a banking crisis on GDP

\begin{tabular}{|c|c|c|c|c|c|c|}
\hline Years after crisis $(k)$ & (1) & (2) & (3) & (4) & (5) & (6) \\
\hline 0 & $0.018 * * *$ & $0.018 * * *$ & $0.018 * *$ & 0.012 & $0.018 * * *$ & $0.018 * * *$ \\
\hline 1 & $0.052 * * *$ & $0.038 * * *$ & $0.034 * * *$ & 0.055 & $0.050 * * *$ & $0.033 * * *$ \\
\hline 2 & $0.084 * * *$ & $0.073 * * *$ & $0.067 * * *$ & 0.067 & $0.075 * * *$ & $0.062 * * *$ \\
\hline 3 & $0.096 * * *$ & $0.085 * * *$ & $0.078 * * *$ & $0.096^{*}$ & $0.076 * * *$ & $0.067 * * *$ \\
\hline 4 & $0.110 * * *$ & $0.099 * * *$ & $0.091 * * *$ & $0.112 *$ & $0.079 * * *$ & $0.074 * * *$ \\
\hline 5 & $0.112 * * *$ & $0.100 * * *$ & $0.091 * * *$ & $0.108 *$ & $0.068 * * *$ & $0.066^{* * *}$ \\
\hline 6 & $0.119 * * *$ & $0.110 * * *$ & $0.100 * * *$ & $0.115 *$ & $0.063 * * *$ & $0.069 * * *$ \\
\hline 7 & $0.140 * * *$ & $0.134 * * *$ & $0.123 * * *$ & $0.143 *$ & $0.069 * * *$ & $0.084 * * *$ \\
\hline 8 & $0.124 * * *$ & $0.109 * * *$ & $0.092 * *$ & $0.137 *$ & $0.041 * *$ & 0.051 \\
\hline 9 & $0.119 * * *$ & $0.110 * * *$ & $0.091 * *$ & 0.128 & 0.025 & 0.047 \\
\hline 10 & $0.096^{* *}$ & $0.089^{*}$ & 0.066 & 0.098 & 0.002 & 0.021 \\
\hline \multicolumn{7}{|c|}{ Dependent variable: $\log$ GDP } \\
\hline Level/growth rate & Level & Gr. rate & Gr. rate & Level & Level & Gr. rate \\
\hline Lags of dep. var. & Yes & Yes & Yes & No & Yes & Yes \\
\hline Banking crisis leads & Yes & Yes & Yes & $\mathrm{n} / \mathrm{a}$ & No & No \\
\hline Coefficient restrictions & No & Yes & No & No & No & No \\
\hline
\end{tabular}

Notes: Column 1: the corrected local projections estimates based on equation (8); columns 2, 3: the same, estimated for GDP growth rates, restricted and unrestricted specifications, respectively; column 4: estimates based on equation (9); column 5: the uncorrected local projection estimates based on equation (6); column 6: the same, estimated for GDP growth rates.

All specifications include country fixed effects. Specifications in levels of GDP include a time trend.

Each specification includes four lags of log GDP (or its growth rate) and of the banking crisis dummy $\left(\begin{array}{lll}R & L & 4\end{array}\right)$.

These lag restrictions were tested and passed by the data for all lengths of the forecast horizon, $p$ values ranging from 0.124 to 0.75 . From this table onwards, asterisks indicate estimates significant at $* * * 1 \%$, $* * 5 \%$ and $* 10 \%$ levels.

Within group serial correlation robust standard errors for the local projections IRF estimates were calculated using option vce (cluster id) in Stata.

\section{EMPIRICAL ESTIMATES OF THE IRFS}

\subsection{Data}

The data on banking crises during the years 19742001 come from Caprio and Klingebiel's (2003) study. Penn World Tables (Heston et al., 2006) provide GDP data from 1960 onwards. The complete data are available for a panel of 99 countries. The average length of a time series depends on the forecast length and ranges from 24 to 15 observations (10-year-ahead forecast). Our sample contains 89 banking crises episodes, so the frequency of observing a banking crisis is 0.036 . The majority of countries (56) experiences this rare event only once, 28 countries had no crisis, 13 countries had two, one country had three and another one had four banking crises. There is a negative correlation $(r=-0.35)$ between the frequency of banking crises and a country's GDP level. Our assumption that the likelihood of a banking crisis is time-invariant is not rejected by the data, as there is no significant time trend in their frequency.

\subsection{Regression Results}

Table I reports estimates of the IRFs to a banking crisis from six different specifications, in levels of log GDP as well as in GDP growth rates. The specification in growth rates comes in two versions: unconstrained; and with the restriction $\Sigma_{r} \delta_{1 r k}=1$ on the coefficients in the underlying equation (8) as nonstationarity of GDP would imply (this restriction is not supported by the data most of the times). We estimate the IRF in all specifications for up to $k=10$ years after the occurrence of a banking crisis. ${ }^{7}$

\footnotetext{
7 The reason for stopping at 10 years is that a local projections estimator of $\operatorname{IRF}(k)$ requires additional $k$ lags of data, depleting the effective sample as $k$ becomes large.
} 
The first four columns in Table I show the results from the specifications that produce unbiased IRF estimates. The results from our preferred specification (the corrected local projections estimator, column 1) show that an average banking crisis leads to GDP losses to the tune of $8 \%$ in the first 2 years after its occurrence. The loss in GDP continues to increase for the next 5 years, reaching a maximum of $14 \% 7$ years after the start of the crisis. The recovery begins only in the eighth year but is too weak to bring GDP back to its pre-crisis level, leaving a 9.6\% loss 10 years after the crisis. These IRF estimates are plotted in Figure 6 together with their 95\% confidence interval, which widens for longer forecast horizons, as expected, reflecting increasing uncertainty in the long-term effects of banking crises.

The estimates from the three other unbiased specifications (columns 24 ) are similar to our preferred one. Comparing these estimates with one another helps gauge the importance of the Nickell bias to our IRF estimates. Applying the corrected local projections estimator to GDP growth rates (columns 2 and 3) exacerbates the bias and hence the its consequences to the IRF estimates, as compared to GDP levels. Indeed, the growth rates-based IRFs are somewhat lower that level-based ones, predicting a $6.68 .9 \%$ GDP loss 10 years after the crisis. On the other hand, recursively eliminating lags of GDP, as is done in the estimator underlying the results in column 4 (equation (9)), reduces the extent of the Nickell bias and its consequences, resulting in a higher estimated GDP loss of 9.8\%. However, given the length of the forecast horizon and the uncertainty in the IRF estimates, the difference in the IRF estimates more and less affected by the Nickell bias is not large.

Columns 5 and 6 report estimation results for specifications that omit banking crises occurring between $t$ and $t+k$. Comparing these estimates with those from corresponding specifications in columns 1 and 3 reveals the size of the bias due to this omission. It turns out that this bias has a larger effect on the IRF estimates than the Nickell bias does. As it increases with $k$, the estimates from the specifications that do and do not correct for it, though similar for the first few years after the crisis, diverge thereafter in a remarkable accordance with the Monte Carlo results presented in section 2.4. In fact, the difference between the biased and unbiased IRF estimates can be so large as to result in contradicting conclusions with respect to the effect of crisis on GDP: for example, while our preferred estimates imply a 9.6\% GDP loss 10 years after the crisis, the results from the corresponding uncorrected local projections regression (6) in column 5 imply a complete recovery. There is a similar pattern in the differences between the estimates from the uncorrected local projections regression in growth rates without coefficient restrictions $(-0.021$, column 6) and its corrected equivalent $(-0.066$, column 3).

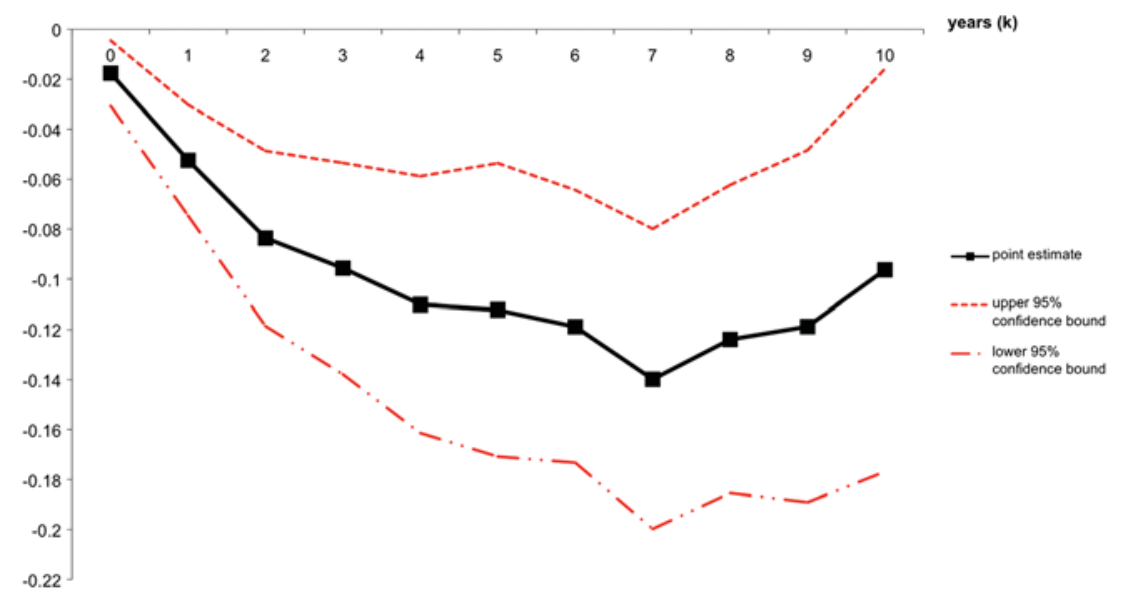

Figure 6. Predicted GDP loss from a banking crisis, and its $95 \%$ confidence interval. Estimates are based on corrected local projections estimator (equation (8)) 
To put the estimated GDP losses due to banking crises into perspective, consider that the median GDP growth rate observed in our sample is roughly $3 \%$ per year, so that the median economy would have grown by $34 \%$ over 10 years. Thus, being linked to a 9.6\% GDP loss over the next 10 years, a single episode of a banking crisis would cost over a quarter (28\%) of the median economy's long-term growth potential. As another illustration of the quantitative importance of banking crises, we calculate the share of residual variance in log GDP explained by these events, and report the results in Table II. The first column in Table II reports the share in the current GDP's residual variance explained by one particular crisis which happened $k$ years ago. The second column contains the shares in residual variance due to the entire history of crises. Starting with $0.2 \%$ in the same year, a single banking crisis can explain up to $2 \%$ of GDP's residual variance in the next several years. The history of the banking crisis during the last 10 years explains just under $9 \%$ of the current (log) GDP's residual variance. For a relatively rare event such as a banking crisis, these shares are rather high.

Having estimated the GDP loss due to a banking crisis on the entire sample, we now repeat the exercise on subsets of countries, to appreciate the role of local context in shaping the response of GDP to a banking crisis. We rerun our corrected local projections estimator (equations (8)) and (9) separately on subsamples of African countries (41 countries) and the rest (54, excluding four transition countries). The results are reported in Table III and Figure 7. African countries suffer more profound GDP losses after a banking crisis (up to 17.5\%) than do other countries (up to 9\%), but experience stronger recovery towards the end of the 10-year period. The IRFs for the African subsample are less precisely estimated, however, suggesting that the effects of banking crises for those countries are also more heterogeneous. Hence the differences in the local context between the two subsamples, such as relatively high political volatility in the African countries, seem to affect GDP's response to banking crises in an important way.

\subsection{The Exogeneity of Banking Crises}

Our method relies on the assumption that banking crises are independent of all realizations of the error term, $\mathrm{E}\left[u_{i, s} d_{i, t}\right]=0, \forall s, t$. In this section, we test the part of this assumption concerning the exogeneity

Table II. Share of residual variance in log GDP explained by banking crises

\begin{tabular}{lcc}
\hline Years after crisis $(k)$ & Single crisis & History of crises \\
\hline 0 & 0.002 & 0.011 \\
1 & 0.005 & 0.019 \\
2 & 0.011 & 0.026 \\
3 & 0.010 & 0.033 \\
4 & 0.012 & 0.046 \\
5 & 0.012 & 0.059 \\
6 & 0.014 & 0.070 \\
7 & 0.020 & 0.077 \\
8 & 0.014 & 0.083 \\
9 & 0.013 & 0.086 \\
10 & 0.009 & 0.089 \\
\hline
\end{tabular}

Note: The share of residual variance explained by banking crises is calculated for each $k$ as the ratio $\frac{r_{1, k}}{r_{2, k}}$, where $r_{1, k}, r_{2, k}$ are residual sums of squares (RSS). For the results in the second column, $r_{1, k}$ is the RSS of the autoregressive model without banking crises:

$$
y_{i, t+k} \quad \delta_{0 i k}+\delta_{0 k}^{*} t+\sum_{r=1}^{R} \delta_{1 r k} y_{i, t-r}+v_{i, t+k}^{1}
$$

and $r_{2, k}$ is the RSS of the augmented local projections equation (8), from which our preferred IRF estimates are derived. For the results in the first column, $r_{2, k}$ is the same, and $r_{1, k}$ is the RSS of equation (8) with all banking crises occurring not in year $t$ are present. This ensures that we capture the share of variance explained by a single banking crisis. 
Table III. Impulse response estimates by subsample of countries

\begin{tabular}{|c|c|c|c|c|}
\hline \multirow[b]{2}{*}{ Years after crisis $(k)$} & \multicolumn{2}{|c|}{ Africa (41 countries) } & \multicolumn{2}{|c|}{ The rest (54 countries) } \\
\hline & Eqn (8) & Eqn (9) & Eqn (8) & Eqn (9) \\
\hline 0 & $0.017 *$ & 0.033 & 0.011 & 0.005 \\
\hline 1 & $0.053 * * *$ & 0.024 & $0.038 * * *$ & 0.022 \\
\hline 2 & $0.092 * * *$ & 0.059 & $0.060 * * *$ & 0.019 \\
\hline 3 & $0.113^{* * * *}$ & 0.106 & $0.062 * * *$ & 0.039 \\
\hline 4 & $0.141 * * *$ & 0.108 & $0.069 * * *$ & $0.057 *$ \\
\hline 5 & $0.137 * *$ & 0.088 & $0.077 * * *$ & $0.091 * * *$ \\
\hline 6 & $0.143^{* *}$ & 0.123 & $0.089 * * *$ & $0.086^{* * * *}$ \\
\hline 7 & $0.175 * * *$ & 0.183 & $0.092 * * *$ & $0.086^{* * * *}$ \\
\hline 8 & $0.157 * *$ & 0.185 & $0.076^{* * *}$ & $0.080 * * *$ \\
\hline 9 & $0.172 * *$ & 0.176 & $0.064 * *$ & $0.059 * *$ \\
\hline 10 & 0.126 & 0.113 & $0.067 * * *$ & $0.069 * * *$ \\
\hline
\end{tabular}

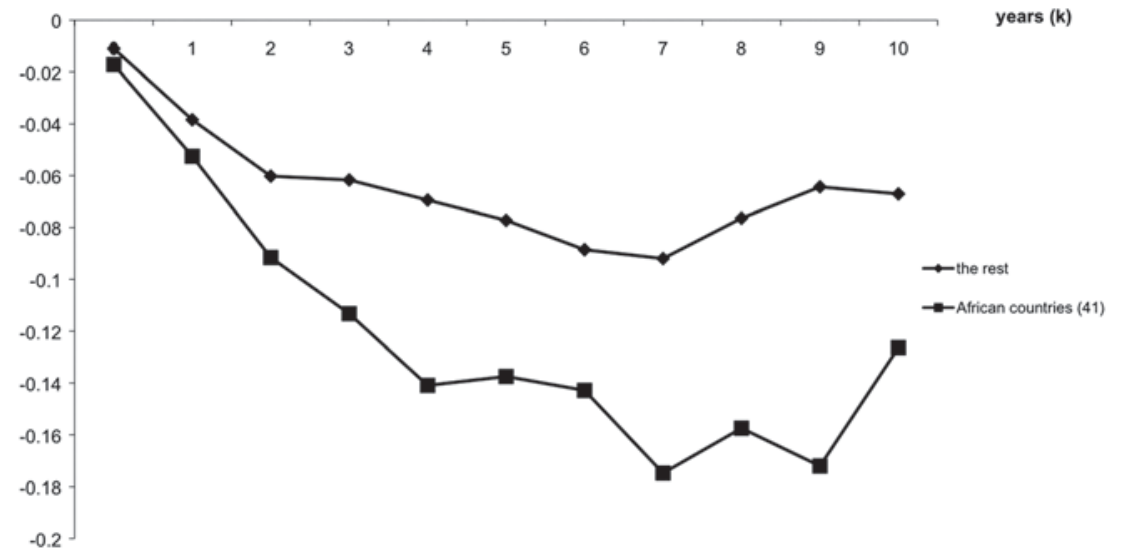

Figure 7. Estimated IFRs by subsample of countries

of banking crises to past realizations of the error term; the exogeneity of banking crises to the error term's future realizations will remain our maintained assumption.

The idea of an expectations bubble typically preceding the crisis, which would give rise to a correlation between past GDP levels and a current banking crisis, was advanced in Jorda et al. (2011). This correlation can have two mechanisms behind it, depending on whether the causality runs from GDP to the banking crisis or in the opposite direction. An episode of excessive GDP growth may create overoptimism in the banking sector, leading to a crisis at the end of it. Alternatively, credit booms that precede banking crises may fuel excess growth through cheap credit, which stops abruptly when the credit boom comes to an end. We test the implications of these mechanisms for the likelihood of a banking crisis as well as for GDP.

To capture the first mechanism, that excessive growth leads to a banking crisis in the future, we run a logit regression of the banking crisis dummy on lagged GDP levels. The results, reported in Table IV, suggest that lagged GDP levels have no predictive power for the likelihood of a banking crisis, giving little support to the idea that excessive growth causes a banking crisis in the future. To test the second mechanism, that credit booms fuel excessive growth, we include in the local projections regression banking crises occurring up to 3 years after the current GDP. The results in Table V, 
Table IV. The likelihood of a banking crisis as a function of past GDP levels

\begin{tabular}{|c|c|c|c|c|}
\hline \multirow[b]{2}{*}{ GDP lag } & \multicolumn{2}{|c|}{ (1) } & \multicolumn{2}{|c|}{ (2) } \\
\hline & Point estimate & Std error & Point estimate & Std error \\
\hline 1 & 1.471 & 1.249 & 1.046 & 1.347 \\
\hline 2 & 0.527 & 2.130 & 0.562 & 2.154 \\
\hline 3 & 1.174 & 2.284 & 1.153 & 2.245 \\
\hline 4 & 0.209 & 1.437 & 0.511 & 1.428 \\
\hline Fixed effects & \multicolumn{2}{|c|}{ No } & \multicolumn{2}{|c|}{ Yes } \\
\hline
\end{tabular}

Note: The estimates are obtained by running a logit regression of the banking crisis dummy on the lags of GDP, with and without country fixed effects.

Table V. GDP growth before the start of a banking crisis

\begin{tabular}{lcc}
\hline Years before crisis $(k)$ & Point estimate & Std error \\
\hline 1 & 0.014 & 0.060 \\
2 & 0.015 & 0.060 \\
3 & 0.026 & 0.057 \\
\hline
\end{tabular}

Note: results are based on specification (9) augmented with leads of the banking crisis dummy as follows:

$$
y_{i, t+k} \quad \gamma_{0 i k}+\gamma_{0 !} t+\sum_{k=-3}^{\infty} \gamma_{2 k} d_{i, t-k}+v_{i, t}
$$

The coefficients reported in the table for 1,2 and 3 years before the crisis are those on $\gamma_{2}, 1, \gamma_{2}, 2$ and $\gamma_{2}, \quad 3$, respectively.

based on equation (9), show no significant link between future banking crises and current GDP. Thus past GDP and banking crises are unrelated.

\section{CONCLUDING REMARKS}

We began with Jorda's (2005) local projections estimator of IRFs, which, unlike the analytical estimator, calculates an IRF from a separate regression for each forecast horizon. This estimator is less efficient than the analytical estimator, butis more robust to model misspecifications, especially for longer forecast horizons. This advantageous property has been illustrated with two examples, the second one linked to a particularly relevant case of the misspecification that Cai and Den Haan (2009) call the 'one-type-shock' model. Both examples have shown superior performance of the local projections estimator, albeit at the cost of some efficiency loss.

Yet the main contribution of our study to the literature lies in showing a so far unnoticed bias in the local projection estimator, and in offering a simple solution to correct this bias. The bias occurs due to this estimator's failure to use the information on the crises occurring within the forecast horizon. We show that even if the crisis occurs only once in the history of observations, the local projections regression will omit it for some ts. This omission will result in its effect on GDP being absorbed in the regression's fixed effect, causing a bias to it which will translate one-to-one into the bias of the IRF estimates. We provide an analytical expression for the size of this bias and show, in particular, that it increases with the length of forecast horizon. Our proposed correction involves augmenting the local projections equation with banking crises occurring within the forecast horizon. Our simulation results 
show its effectiveness and robustness even under severe misspecifications of the underlying dynamic model for GDP. Situations giving rise to the local projections bias occur in a variety of settings. Therefore, our method is widely applicable.

Applying our method to the banking crisis data, we find that the GDP loss after a banking crisis is large, standing at just under $10 \% 10$ years after the crisis. The consequences of banking crises vary by country group. We find that in African countries banking crises are more severe, costing 11 13\% of GDP over a 10-year period after the crisis, whereas in other countries the loss is smaller, about $7 \%$ over the same period. Relating our findings to Cerra and Saxena's (2008), who used the analytical method of calculating the IRF, there are two differences. First, our full-sample estimate of the banking crisis's output effect over 10 years $(9.6 \%)$ is larger than theirs $(7.5 \%)$. Second, their estimate of the long-term GDP loss for African countries (6\%) is smaller than for high-income countries (15\%), whereas our finding is the opposite. While it is difficult to pinpoint the exact source of these differences, the uniformity of their dynamic model's structure coupled with the analytical IRF estimator's sensitivity to model misspecification might be one explanation. Qualitatively, however, our results are similar to theirs, implying that after-crisis GDP is unlikely to return to its pre-crisis path in the foreseeable future.

\section{ACKNOWLEDGEMENTS}

The authors thank Adam Elbourne, Wouter den Haan, Oscar Jorda, Bert Smid and the participants of 2010 Canadian Economics Association conference for comments on previous versions of this paper.

\section{REFERENCES}

Alvarez J, Arellano M. 2003. The time series and cross section asymptotics of dynamic panel data estimators. Econometrica 71(4): 11211159.

Cai X, Den Haan W. 2009. Predicting recoveries and the importance of using enough information. Working paper. Available: http://econ.lse.ac.uk/staff/wdenhaan/papers.htm [10 June 2011].

Campbell JY, Mankiw NG. 1987. Are output fluctuations transitory? Quarterly Journal of Economics 102(4): 857880.

Caprio G, Klingebiel D. 2003. Episodes of systemic and borderline financial crises. Available: http://go. worldbank.org/5DYGICS7B0 [19 May 2013].

Cerra V, Saxena SC. 2008. Growth dynamics: the myth of economic recovery. American Economic Review 98(1): 439457.

Chong Y, Jorda O, Taylor AM. 2012. The Harrod Balassa Samuelson hypothesis: real exchange rates and their long run equilibrium. International Economic Review 53(2): 609634.

Faust J, Wright JH. 2011. Efficient prediction of excess returns. The Review of Economics and Statistics 93(2): 647659.

Heston A, Summers R, Aten B. 2006. Penn World Table Version 6.2. Center for International Comparisons of Production, Income and Prices at the University of Pennsylvania.

Jorda O. 2005. Estimation and inference of impulse responses by local projections. American Economic Review 95(1): 161182.

Jorda O, Schularick M, Taylor AM. 2011. When Credit Bites Back: Leverage, Business Cycles, and Crises. NBER working paper 17621.

Judson RA, Owen AL. 1999. Estimating dynamic panel data models: a guide for macroeconomists. Economics Letters 65(1): 915.

Mood A, Graybill F, Boes D. 1974. Introduction to the Theory of Statistics. McGraw Hill: New York.

Nickel S. 1981. Biases in dynamic models with fixed effects. Econometrica 49(6): 14171426. 